This item was submitted to Loughborough's Research Repository by the author.

Items in Figshare are protected by copyright, with all rights reserved, unless otherwise indicated.

\title{
Low correlation multiple antenna system for mobile phone applications using novel decoupling slots in ground plane
}

PLEASE CITE THE PUBLISHED VERSION

http://dx.doi.org/10.1109/LAPC.2013.6711966

\section{PUBLISHER}

(c) IEEE

\section{VERSION}

AM (Accepted Manuscript)

\section{PUBLISHER STATEMENT}

This work is made available according to the conditions of the Creative Commons Attribution-NonCommercialNoDerivatives 4.0 International (CC BY-NC-ND 4.0) licence. Full details of this licence are available at: https://creativecommons.org/licenses/by-nc-nd/4.0/

\section{LICENCE}

CC BY-NC-ND 4.0

\section{REPOSITORY RECORD}

Saraereh, Omar A., C.J. Panagamuwa, and J.C. Vardaxoglou. 2019. "Low Correlation Multiple Antenna System for Mobile Phone Applications Using Novel Decoupling Slots in Ground Plane”. figshare. https://hdl.handle.net/2134/15820. 


\section{Low Correlation Multiple Antenna System for Mobile Phone Applications Using Novel Decoupling Slots in Ground Plane}

\author{
Omar A. Saraereh \\ Department of Electrical Engineering \\ The Hashemite University \\ Zarqa, Jordan \\ eloas2@hu.edu.jo
}

\author{
C. J. Panagamuwa and J. C. Vardaxoglou \\ School of Electronic, Electrical and Systems Engineering \\ Loughborough University \\ Loughborough, UK \\ c.j.panagamuwa@lboro.ac.uk
}

\begin{abstract}
A compact low profile multiple antenna system for multiple-input-multiple-output (MIMO) applications is proposed. The antenna system combines two monopole type printed antennas with a slotted ground plane for low correlation and high isolation characteristics. The main antenna covers the twelve wireless communication bands required for LTE, GSM, UMTS2110, Bluetooth, WiMAX and WLAN. The auxiliary antenna has a very small volume compared to the main one and covers the ultra-wideband (UWB) frequency range (3.74-12 $\mathrm{GHz}$ ). The antennas are positioned at opposite ends of the system's ground in order to reduce the mutual coupling between them. The isolation maintained is better than $20 \mathrm{~dB}$ over the desired frequency bands, resulting in an envelope correlation coefficient of less than 0.08 . The simulation results show good $\mathrm{S}$ parameters, high gain and radiation efficiency, and relatively stable radiation patterns. Due to the compact size and the ultrawide bandwidth, the proposed multiple antenna system is suitable for communication handsets that have size limitations. Results are presented and discussed.
\end{abstract}

Keywords-mobile antenna; MIMO; correlation; mutual decoupling; LTE; planar monopole; slotted ground; multiband.

\section{INTRODUCTION}

The rapid growth in wireless communication systems has proportionally led to the advancement of antenna systems for diversity and multiple-input multiple-output (MIMO) handset applications [1, 2]. Multiple antenna systems enable high quality and high capacity gain communications using Long Term Evolution (LTE) and Wireless Wide Area Network (WWAN) mobile networks without the need for additional power or spectrum [3]. However, it is a challenging task for antenna designers to place multiple antenna system within the limited space since high performances requires high isolation and decoupling between antenna elements and even the radiating ground plane $[4,5]$. Different techniques have been introduced in the literature to reduce mutual coupling in closely located antennas in MIMO systems [6-10]. Among these techniques, inserting decoupling slots into the system ground plane as proposed in [10] is a favourable technique. The proposed design in [10] does not cover the low frequency bands LTE700/GSM850/GSM900 due to their strong mutual coupling.

In this paper, a novel multiple antenna system for mobile handset applications is proposed. It covers standards LTE700/2300/2500, GSM 850/900/1800/1900, UMTS2110, ISM/Bluetooth, WiMAX 2500/3500, WLAN2400/5200/5800 and UWB in the frequency range $(3.74-12 \mathrm{GHz})$ at return loss better than $6 \mathrm{~dB}$ simultaneously. The antennas can also be used for MIMO operations.

\section{MULTIPLE ANTENNA SYSTEM STRUCTURE}

The proposed multiple antenna system design compromising two antennas are denoted through this paper as main antenna and auxiliary antenna. Both encompass coupledfed elements and monopole type antennas. Fig. 1(a), (b) and (c) show the geometry details and dimensions of the proposed antenna system. The presented antenna arrangement consists of main antenna, auxiliary antenna, system PCB (substrate), protruded slotted ground plane and two feeding ports. The main ground is printed on a $0.8 \mathrm{~mm}$ thick FR-4 substrate $\left(\varepsilon_{\mathrm{r}}=4.4\right.$ and loss tangent is 0.02$)$ of total size $50 \times 120 \mathrm{~mm}^{2}$. The dimensions of the PCB and the ground size considered here are practical for general smart phones or personal digital assistant (PDA) phones that are available in the market.

The system ground plane is printed on the back side of the circuit board, leaving a no-ground portion of a size $14.6 \times 50 \mathrm{~mm}^{2}$ at the upper region of the PCB, see Fig. 1(b). The main antenna elements occupy $17 \times 50 \mathrm{~mm}^{2}$ on the substrate front side as shown in Fig. 1(a). At the lower region of the PCB, the ground has been protruded to obtain different coupling elements: L-shape with a circular section at the corner, J-shape with three segments, square open loop shape with $13 \mathrm{~mm}$ side and no-ground portion of $8.7 \times 9.5 \mathrm{~mm}^{2}$ to align with the auxiliary antenna main radiating element. The auxiliary antenna structure consists of wideband printed planar monopole of $8.7 \times 9.5 \mathrm{~mm}^{2}$ with a semi-circular section.

The main antenna with its coupled feeding structure and slotted ground was introduced by the author in [11]. A $50 \Omega$ coaxial feed line (feeding port 1) was used to excite the main antenna. A shorting pin was included to connect the shorted 
coupling strip (parasitic element) to the underlying ground region of the PCB. In order to broaden the bandwidth of the main antenna, different slots and notches were introduced into the system ground plane underneath the lower edge of the main antenna. More details of this coupled-fed main antenna and the slotted ground plane with full dimensions can be found in [11].

This presented paper adds an auxiliary antenna in the form of compact wideband folded monopole antenna with triple resonant modes to that of [11] for UWB operation and to perform MIMO operation with the main antenna. The auxiliary antenna structure has been coupled with the J-shape and L-shape coupling elements protruding from the ground plane as shown in Fig. 1. The inclusion of the auxiliary antenna has a detrimental effect on the impedance bandwidth of some of the main antenna bands. The J-shape has been introduced in the ground plane to counteract this reduction in impedance bandwidth. The auxiliary antenna main radiating element is feed by a $50 \Omega$ coaxial feed line (feeding port 2 ).

The last part in the proposed multiple antenna system is the decoupling filter which has been inserted into the system ground plane as shown in Fig. 1(b). It reduces strong mutual coupling between main and auxiliary antenna, mainly at the lower frequency bands. The decoupling filter arrangement includes four groups of slots with different dimensions, see Fig. 1(c):

- group1 consists of two series identical slots parallel to a third smaller one,

- group2 has three parallel identical slots,

- group3 in the form of U-shape slots with sleeves and Tshape slot at its symmetry axis

- group4 as an L-shape slots adjacent to feeding port 2.

The location and dimensions of the slots compromising suggested groups affect the isolation between the antennas, especially at the lower resonant frequency bands. Over the upper bands, the effect of the slots is slight because the electrical length of the distance between the two ports is larger. By optimising the location and dimensions of slots, the mutual coupling between the main and auxiliary antennas has been decreased significantly, as well as improving the Sparameters. Through the design process for the decoupling filter groups, parametric studies revealed that positioning the groups closer to the main antenna degrades the lower frequency bands significantly and increases mutual coupling between antennas. The aforementioned reason explains why these groups are positioned very close to the auxiliary antenna. The closest group to the auxiliary antenna feeding port is group4 (L-shape slots) which has the strongest effect on reducing coupling and simultaneously maintaining good Sparameters.

The plastic housing cover is also considered for the real mobile terminal situation. The plastic material utilised is ABS polymer $\left(\varepsilon_{\mathrm{r}}=3.3\right.$ and loss tangent 0.02$)$. The housing cover has dimensions of $54 \times 130 \mathrm{~mm}^{2}$ with $1 \mathrm{~mm}$ thickness. It also has a $3 \mathrm{~mm}$ gap between the antenna and the inner housing cover.
The selected dimensions allow the system circuit board to be at the centre of the plastic housing.

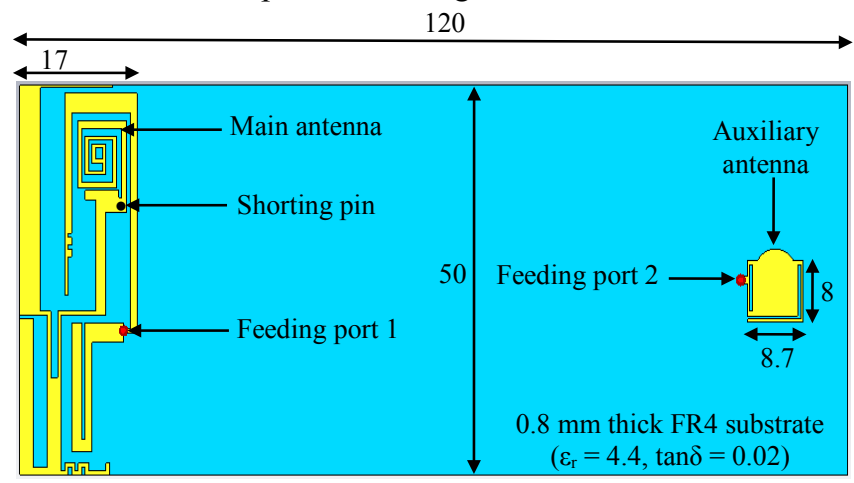

(a) Top view for main and auxilairy antennas

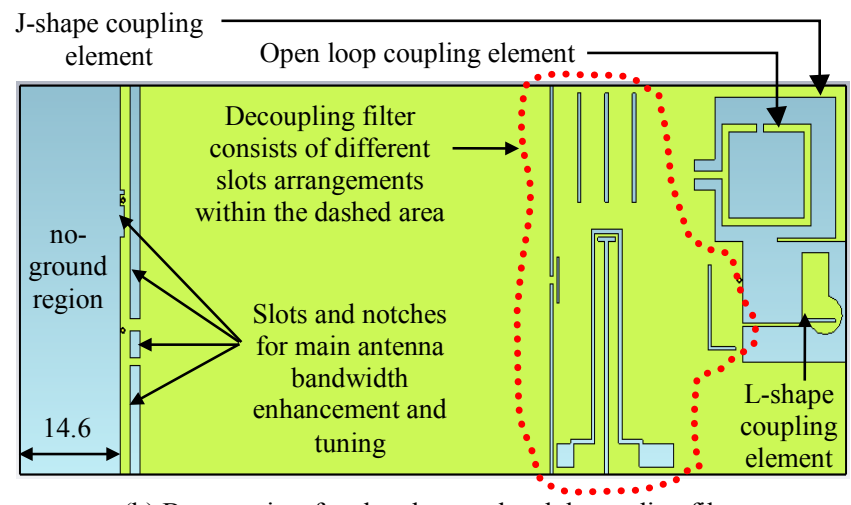

(b) Bottom view for sloted ground and decoupling filter

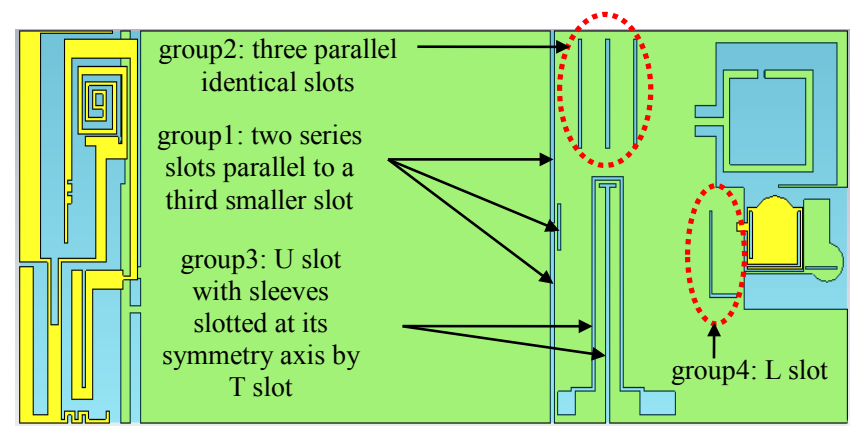

(c) Top view with the bottom layer showing through

Fig. 1. Prposed multiple antenna system structure and dimensions (mm).

\section{RESULTS, ANALYSIS AND DISCUSSION}

Fig. 2 shows the simulated S-parameters $\left(\mathrm{S}_{11}, \mathrm{~S}_{21}, \mathrm{~S}_{12}\right.$ and $\mathrm{S}_{22}$ ) for the proposed multiple antenna system before inserting the decoupling filter into the ground plane. Simulations have been carried out using the electromagnetic computer-aided simulator CST Microwave Studio ${ }^{\text {TM }}$. The $S_{11}$ results show that the impedance bandwidth of the main antenna offers a lower band at resonant frequency $0.735 \mathrm{GHz}$ which requires widening in order to cover LTE700 and GSM850/900. The second frequency band resonates at $2.4 \mathrm{GHz}$ to cover GSM1800/1900, UMTS (1920-2170 MHz), LTE2300 (2305$2400 \mathrm{MHz})$, WLAN2400 (2400-2484 MHz). However, this antenna system only covers $50 \%$ of LTE2500 operating band 
and therefore also requires improving. The third band resonates at $3.538 \mathrm{GHz}$ to cover WiMAX3500 and the fourth band resonates at $5.353 \mathrm{GHz}$ to cover WLAN5200/5800. The simulated $\mathrm{S}_{21}$ and $\mathrm{S}_{12}$ of the multiple antenna system without decoupling filter structures are approximately identical and show isolation better than $13 \mathrm{~dB}$ over the entire frequency range of interest as shown in Fig. 2. The reverse reflection coefficient $\mathrm{S}_{22}$ of the auxiliary antenna shows UWB operation ranges from $3.889 \mathrm{GHz}$ to $12 \mathrm{GHz}$ (return loss better than 10 dB).

The simulated S-parameter results obtained by adding the decoupling filter to the ground plane are shown in Fig. 3. It can be seen clearly from the $S_{11}$ curve that the main antenna lower band has been improved significantly to cover LTE700 and GSM850/900 using an impedance bandwidth in the frequency range $(0.674-1.089 \mathrm{GHz})$. The main antenna with the aid of the proposed decoupling filter is able to cover more than twelve standard operating bands for LTE, GSM, UMTS, WiMAX, and WLAN for mobile communications and several frequency bands above $6.5 \mathrm{GHz}$ with isolation better than 20 $\mathrm{dB}$ in the bands of interest. The auxiliary antenna reflection coefficient $\mathrm{S}_{22}$ curve with the decoupling filter shows three wideband resonant modes as shown in Fig. 3. The three operating bands for the auxiliary antenna are band 1 (1.972$3.087 \mathrm{GHz}$ ) covering LTE2300/2500, and WLAN2400, band $2(3.740-7.227 \mathrm{GHz})$ covering WLAN5200/5800 and band 3 (7.848-12 GHz) for UWB applications.

One of the common methods to approve the performance of the presented design for multiple port applications is calculating the envelope correlation coefficient (ECC) in terms of S-parameters of the antenna system by using the following equation $[12,13]$ :

$$
\rho_{e}(i, j, N)=\frac{\left|\sum_{n=1}^{N} S_{i, n}^{*} S_{n, j}\right|^{2}}{\prod_{k=(i, j)}\left[1-\sum_{n=1}^{N} S_{i, n}^{*} S_{n, k}\right]}
$$

where $\mathrm{N}$ is the number of antenna comprising the system. The used equation is very simple and does not give precise information about the coupling between the antennas since it does not include both scattering parameters and the intrinsic power losses in the radiating structures simultaneously [14]. It should be emphasized here that the decoupling filter is resonating and plays an important role in covering the lower bands due to the fact that the entire structure resonating before even adding the auxiliary antenna.The calculated envelope correlation coefficient (ECC) to measure the diversity gain of the proposed multiple antenna system is plotted in Fig. 4. Fig 4(a) shows the effect of inserting each decoupling group $(1,2$, 3 and 4) one by one on the ECC in the lower frequency bands. Rigorous parametric studies have led to optimized dimensions and positions for the slots to result in lowest coupling and good S-parameter performance, simultaneously, in the lower

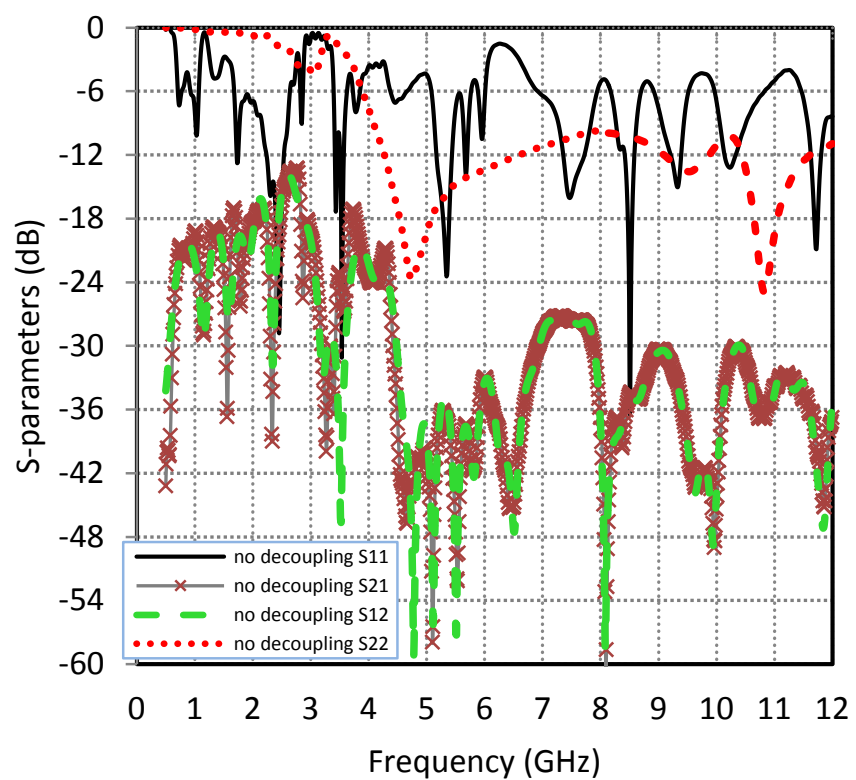

Fig. 2. Simulted S-parameters of main and auxilary antennas without decoupling filter.

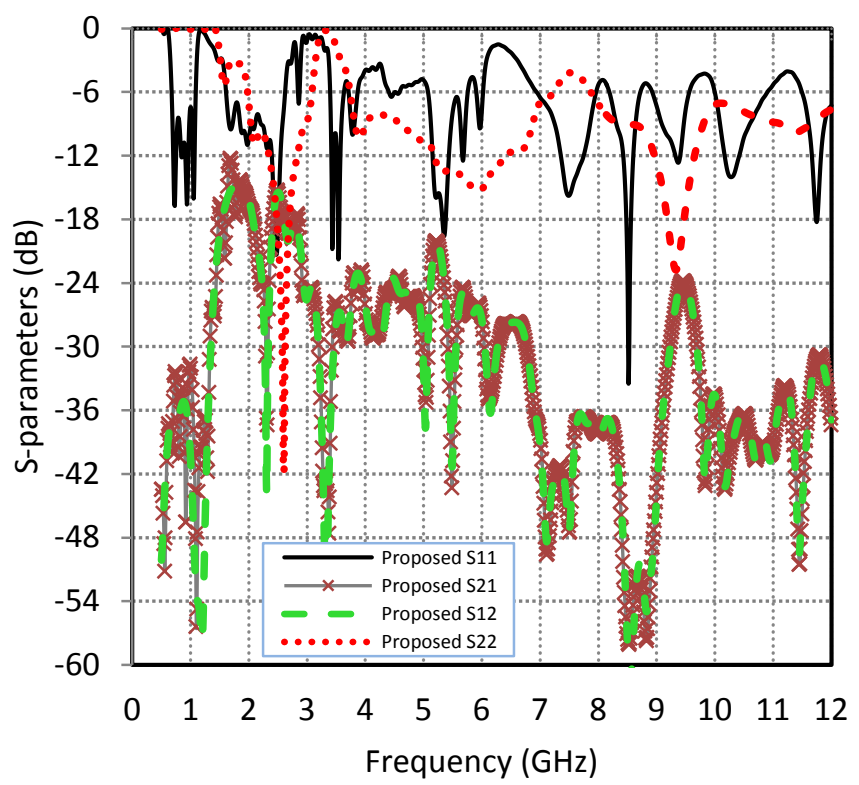

Fig. 3. Simulated S-parameters of the proposed multiple antenna system with decoupling filter.

bands. The optimal length is found to be about $\lambda \backslash 4$ in the lower bands. In addition, the slots are reused as parasitic elements at higher frequencies. Fig. 4(b) shows ECC comparison with and without the existence of the presented decoupling technique over the entire frequency range $(0.5-12 \mathrm{GHz})$. It is obvious from the curves how introducing the decoupling filter into the ground plane system reduces significantly the ECC from 0.98 to less than 0.3 for the entire frequency range. The proposed design has an ECC less than 0.08 in the desired operating bands, which means that the proposed multiple antenna system has good diversity gain. The introduced decoupling filter slots block and suppress the current flowing from the excited Port 1 
to the coupled excited Port 2 and vice-versa. .Fig. 5 displays the surface current distributions at $0.715 \mathrm{GHz}$ with and without decoupling filter. The decoupling filter slots' electrical lengths have significantly improved the impedance bandwidth and reduced the ECC from 0.9 to 0.08 .

The proposed multiple antenna system efficiency is presented in Fig. 6. It can be noted that, over the frequency bands under consideration, both main and auxiliary antennas radiated efficiencies are about 90-98\%. The achieved radiated efficiencies results are significant for practical mobile devices and internet applications. Fig. 7 shows the radiation characteristics of the proposed multiple antenna system at $0.715 \mathrm{GHz}$ for main antenna and at $10 \mathrm{GHz}$ for the auxiliary antenna. The antenna system exhibits omnidirectional radiation characteristics for low frequencies and more variations in the radiation patterns are observed at high frequencies. Generally, most of radiation patterns are nearly omnidirectional with some nulls.

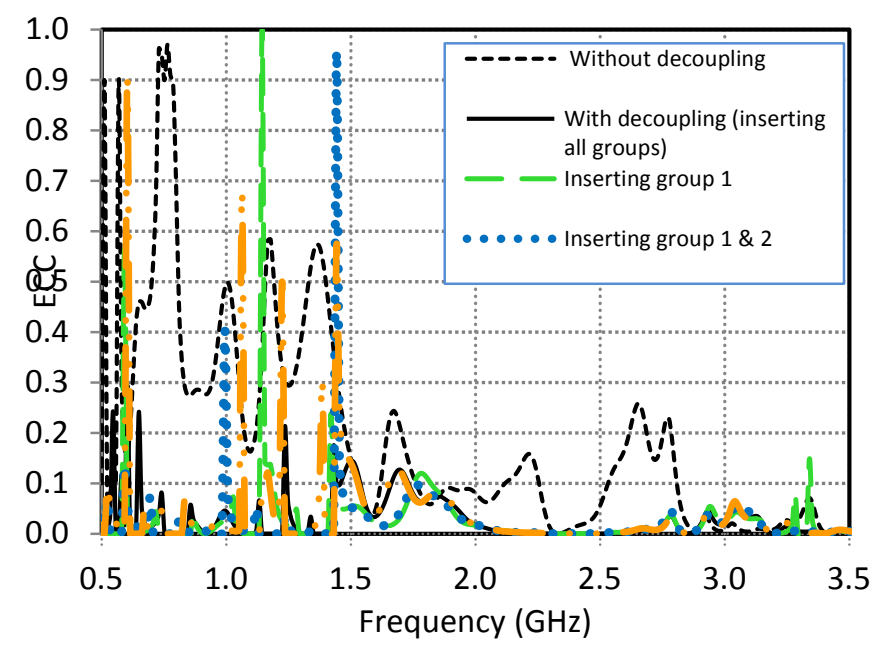

(a)

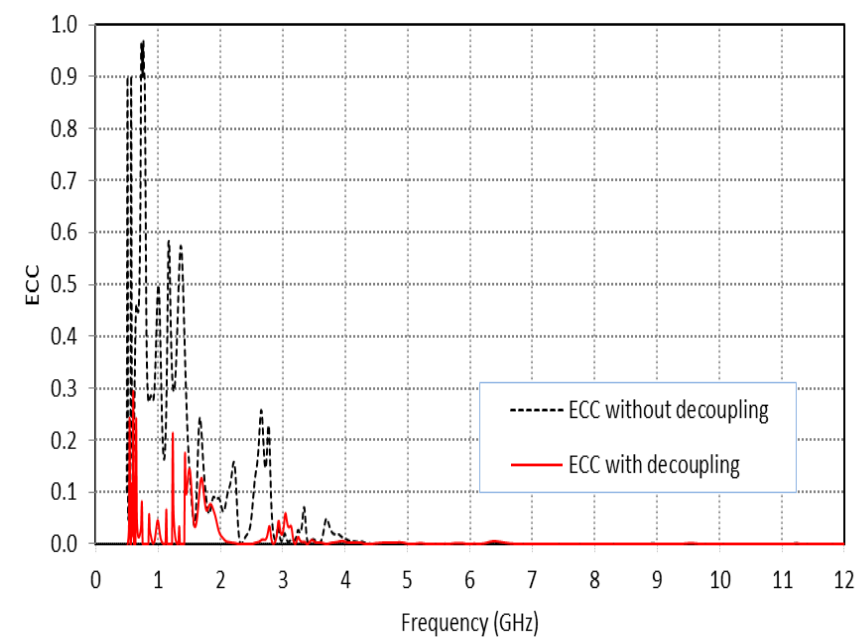

(b)

Fig. 4. Calculated results of enevelope correlation coeffiect: (a) for different proposed groups in the lower frequency bands (b) with and without proposed decoupling filter over frequency range $(0.5-12 \mathrm{GHz})$.
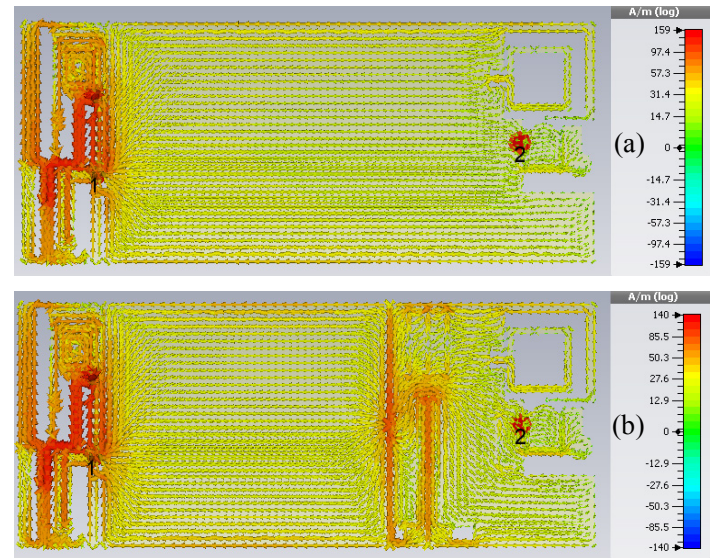

Fig. 5. Surface current distributions of the proposed multiple antenna system (a) without decoupling filter and (b) with decoupling filter at $0.715 \mathrm{GHz}$.

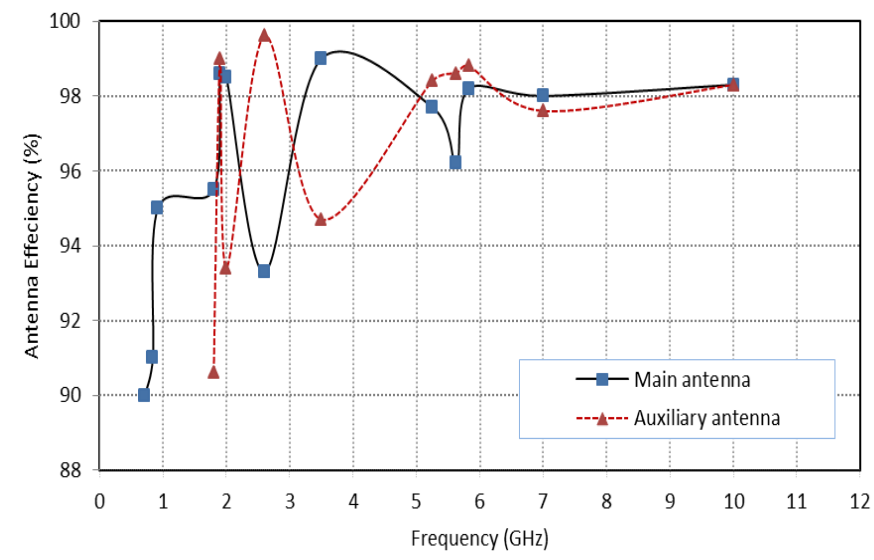

Fig. 6. Radiated antenna effeciency for main and auxiliary antennas.

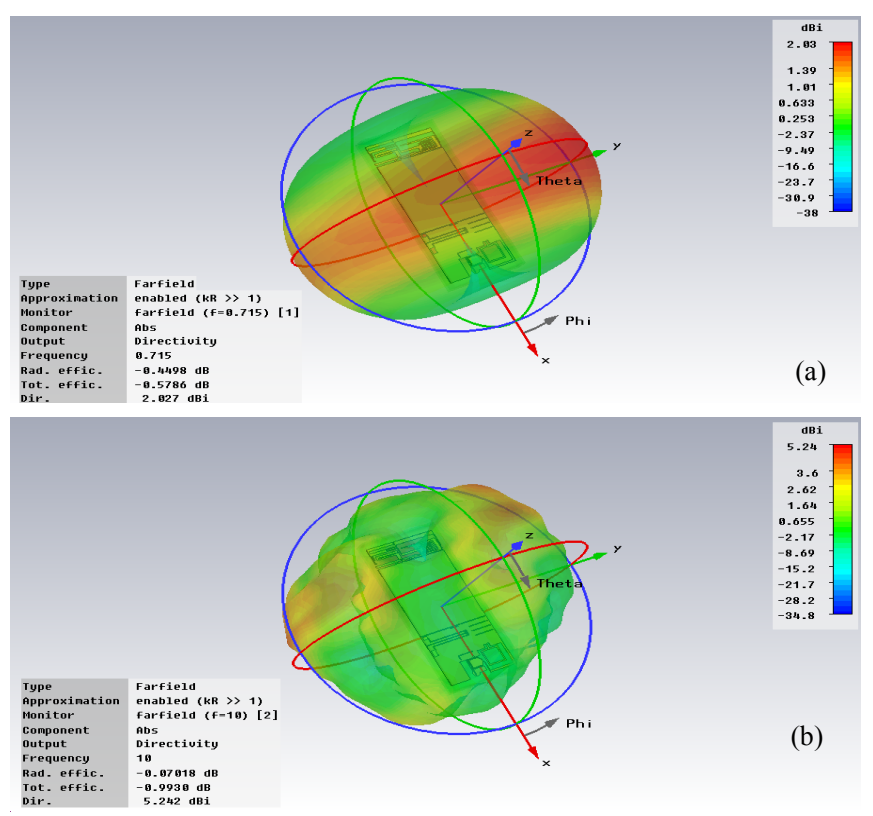

Fig. 7. Radiated characterstics of the proposed multiple antenna system for (a) main antenna at $0.715 \mathrm{GHz}$ (b) auxiliary antenna at $10 \mathrm{GHz}$. 


\section{CONCLUSION}

This article has proposed a novel multiple antenna system with an intelligent decoupling technique for high isolation better than $20 \mathrm{~dB}$. The system consists of two planar printed compact monopole antennas with a decoupling filter among the system ground plane to accomplish ECC below 0.08 over the entire frequency bands. Antenna performances with and without the proposed decoupling filter have been listed. The proposed design covers all the standard operating bands in the frequency range $0.5-12 \mathrm{GHz}$ for LTE, GSM, UMTS, ISM, WiMAX, WLAN and UWB for both mobile and internet applications (return loss better than $6 \mathrm{~dB}$ ). The radiation patterns and efficiencies of the design make it a potential candidate for mobile device applications. The presented decoupling filter design can be can be applied to different multiple antenna or MIMO systems.

\section{REFERENCES}

[1] D. Gesbert, M. Shafi, D. S. Shiu, P. Smith, and A. Naguib, "From theory to practice: An overview of MIMO space-time coded wireless systems," IEEE J. Sel. Areas Commun., Vol. 21, No. 3, pp. 281-302, Apr. 2003.

[2] S. Cui, Y. Liu,W. Jiang, S. X. Gong, Y. Guan, and S. T. Yu, "A novel compact dual-band MIMO antenna with high port isolation," J. of Electromagn. Waves and Appl., Vol. 25, pp. 1645-1655, June 2011.

[3] W. Kin-Lu, K. Ting-Wei, and Tu. Ming-Fang, "Internal mobile phone antenna array for LTE/WWAN and LTE MIMO operations," Microwave and Opt Tech Letters., Vol. 53, No. 7, July 2011.

[4] M. A. Jensen, and J.W. Wallace, "A review of antennas and propagation for MIMO wireless communications," IEEE Trans. Antennas Propag., Vol. 52, No. 11, pp. 2810-2824, Nov. 2004.
[5] E. K Antonino-Daviu, M. Cabedo-Fabres, B. Bernardo-Clemente, and M. Ferrando-Bataller, "Printed multimode antenna for MIMO systems," J. of Electromagn. Waves and Appl., Vol. 25, pp. 2022-2032, March, 2011.

[6] F. Yang, and Y. R. Samii, "Microstrip antennas integrated with electronic band gap EBG structure," IEEE Trans. Antennas Propag., Vol. 51, No. 10, pp. 2036-3946, Oct. 2003.

[7] S. Cui, S. X. Gong, Y. Liu, W. Jiang, and Y. Gaun, "Compact and low coupled monopole antennas for MIMO system applications," J. of Electromagn. Waves and Appl., Vol. 25, 703-712, March, 2011.

[8] J. Jasper Sweetlin, and T. Anita Jones Mary, "Mutual decoupling in quad band MIMO slotted PIFA for wireless applications," Int J. of Engineering and Science., Vol. 1, No. 2, pp. 303-307, 2012.

[9] K. G. Y. Ding, and Z. Feng, "A novel dual-band printed diversity antenna for mobile terminals," IEEE Trans. Antennas Propag., Vol. 55, No. 7, pp. 2088-2096, Oct. 2007.

[10] X. Zhou, X. Quan, and RongLin Li, "A dual-broadband MIMO antenna system for GSM/UMTS/LTE and WLAN handset," IEEE Antennas Wireless Propag Letters., Vol. 11, pp. 551-554, 2012.

[11] O. A. Saraereh , M. A. Smadi, A. K. S. Al-Bayati, J. A. Ghaeb, and A. Nimrat, " A Novel Low-Profile Coupled-Fed Printed Twelve-band Mobile Phone Antenna with Slotted Ground Plane for LTE/GSM/UMTS/WiMAX/WLAN Operations," IET Microwaves, Antennas \& Propagation. (Unpublished).

[12] S. Blanch, J. Romue and I. Corbella, "Exact representation of antenna system diversity performance from input parameter description," Electronic Letters., Vol. 39, No. 9, pp. 705-707, May. 2003.

[13] A. Foudazi, A. Mallahzadeh and S. M. A. Nezhad, "A triple-band WLAN/WiMAX printed monopole antenna for MIMO applications," Microwave and Opt Tech Letters.,Vol. 54, No. 5, pp. 1321-1325, May. 2012.

[14] J. Thaysen and K. B. Jakobsen, "Envelope correlation in (N,N) mimo antenna array from scattering parameters," Microwave and Opt Tech Letters, vol. 48, no. 5, pp. 832-834, 2006. 